\title{
MEDICIÓN DE NIVELES DE PARATHORMONA COMO PREDICTOR DE HIPOCALCEMIA SINTOMÁTICA EN EL POST-OPERATORIO PRECOZ DE TIROIDECTOMÍA TOTAL*
}

\author{
Drs. Gerardo Mordojovich R. ${ }^{1}$, Matías Lavín G. ${ }^{1}$, Nicolás Ávalos J. ${ }^{1,2}$ \\ ${ }^{1}$ Departamento de Cirugía, Hospital Clínico Fuerza Aérea de Chile (FACH), Universidad de Valparaíso. \\ 2 Instituto Chileno de Cabeza y Cuello. Santiago. \\ Chile.
}

\begin{abstract}
\section{Parathormone levels measurement as predictor of symptomatic hypocalcemia after total thyroidectomy}

Introduction: The most frequent complication after total thyroidectomy is hypocalcemia. It is difficult to predict it. The objective of this paper is determinate if measurement of parathormone 6 hours after total thyroidectomy can predict symptomatic hypocalcemia, and determinate associated factors in the development of this complication. Material and Method: Prospective case series. Patients that underwent total thyroidectomy between 2006 and 2008 in our Hospital. We registered epidemiological data, related surgery factors and measurement of parathormone 6 hours after surgery. Hypocalcemia symptoms were registered. We used statistical analysis considering significant $\mathrm{p}<0.05$. Results: We included 82 patients. Median age was 53.2 years. $79.3 \%$ were female. The average of parathormone 6 hours after surgery was $28.7 \mathrm{pg} / \mathrm{dL}$. Sensibility was $100 \%$, specificity $79.4 \%$, positive predictive value $59.4 \%$, negative predictive value $100 \%$ and accuracy $84.1 \%$ to predict symptomatic hypocalcaemia. A statistical association among levels under the normal base line of parathormone and symptomatic hypocalcemia was detected $(\mathrm{p}<0.0001)$. Relative risk was 4.84. Univariated analysis showed association between hypocalcemia and pre-operative thyroid cancer diagnosis $(\mathrm{p}=0.01)$, cervical dissection $(\mathrm{p}=0.03)$ and level of parathormone $(\mathrm{p}=0.002)$. Multivariated analysis showed that only the level of parathormone associates with hypocalcemia $(p=0.002)$. Conclusion: The measurement of parathormone allows identifying which patients are at risk of presenting symptomatic hypocalcemia after total thyroidectomy.

Key words: Parathormone, thyroid surgery, hypocalcemia.

\section{Resumen}

Introducción: La complicación más frecuente de la tiroidectomía total es la hipocalcemia. Su predicción es difícil. El objetivo de este trabajo es determinar si la medición de parathormona a las 6 h posterior a una tiroidectomía total es un factor que pueda predecir la aparición de hipocalcemia sintomática y determinar

*Recibido el 25 de marzo de 2014 y aceptado para publicación el 25 de junio de 2014.

No hubo apoyo financiero. No hay conflicto de interés.

Correspondencia: Dr. Nicolás Ávalos J.

contacto@cabezaycuello.cl 
los factores asociados al desarrollo de esta complicación. Material y Método: Serie de casos prospectiva. Pacientes intervenidos de tiroidectomía total entre 2006 y 2008 en el Hospital FACH. Se registraron datos epidemiológicos, factores relacionados a la cirugía y la medición de parathormona a las 6 h. Se registraron los síntomas de hipocalcemia. Se utilizó estadística analítica considerando significativo $\mathrm{p}<0,05$. Resultados: Se enrolaron 82 pacientes. La edad media fue 53,2 años. El 79,3\% fue de sexo femenino. El promedio de parathormona a las $6 \mathrm{~h}$ fue $28,7 \mathrm{pg} / \mathrm{dL}$. Se obtuvo sensibilidad 100\%, especificidad 79,4\%, valor predictivo positivo $59,4 \%$, negativo $100 \%$ y precisión $84,1 \%$ para predecir hipocalcemia. Cuando los valores de PTH estaban bajo el margen normal, el riesgo relativo de hipocalcemia sintomática fue 4,84 ( $\mathrm{p}<0,0001)$. El análisis univariado mostró asociación entre hipocalcemia y el diagnóstico pre-operatorio de cáncer $(\mathrm{p}=0,01)$, la disección cervical $(\mathrm{p}=0,03)$ y el nivel de parathormona a las $6 \mathrm{~h}(\mathrm{p}=0,002)$. El análisis multivariado demostró que sólo el nivel de parathormona se asocia con hipocalcemia $(\mathrm{p}=0,002)$. Conclusión: La medición de parathormona es un elemento que permite estimar de manera adecuada qué pacientes están en riesgo de presentar hipocalcemia sintomática en el post-operatorio precoz de tiroidectomía total.

Palabras clave: Parathormona, cirugía tiroidea, hipocalcemia.

\section{Introducción}

La tiroidectomía total es frecuente en Chile. $\mathrm{Su}$ prevalencia no está estimada, sin embargo, se espera un incremento en el número de éstas dado al aumento de la tasa de cáncer diferenciado de tiroides a nivel mundial. Las complicaciones propias de la cirugía de tiroides son el hematoma compresivo cervical, la lesión de nervios recurrentes laríngeos y la hipocalcemia ${ }^{1-3}$

La hipocalcemia es la complicación más frecuente. Su definición varía de acuerdo a los distintos autores y forma de cuantificarla ${ }^{4,5}$. Algunos evalúan la hipocalcemia de laboratorio, esto es calcio plasmático inferior a $8,6 \mathrm{mg} / \mathrm{dL}$, o ajustado a niveles de albúmina plasmática o calcio iónico, sin embargo, la hipocalcemia clínicamente relevante es aquella asociada a síntomas 6 . Diversas series muestran que la incidencia de hipocalcemia transitoria va desde un $1,6 \%$ hasta un $50 \% 0^{4-7}$, sin embargo, la mayoría de las series la sitúan entre 1 y $30 \% \%^{4,8,9}$. La incidencia de hipocalcemia permanente va desde un $0 \%$ hasta un $10 \%{ }^{10,11}$, con la mayoría de las series que reportan una incidencia entre el $1 \%$ y $2 \%{ }^{1,3,5}$. La hipocalcemia es el principal predictor de estadía hospitalaria prolongada posterior a una tiroidectomía total ${ }^{11-13} \mathrm{y}$ aquí su importancia para predecir quién va requerir suplemento calcio y quién no.

La hipocalcemia en el post-operatorio de una tiroidectomía total puede ocurrir por diversas razones, sin embargo, la principal causa es la insuficiencia paratiroidea $^{2,3,5,6,14}$. Esto se debe a injuria quirúrgica directa, devascularización, edema post-quirúrgico, formación de un hematoma y remoción de una o más glándulas paratiroides ${ }^{1,4,9,15,16}$. Dada su estrecha relación anatómica con la tiroides este daño puede ocurrir al manipular la tiroides ${ }^{17}$, durante la disección cervical central ${ }^{15,17}$ o en la disección de los vasos tiroideos. Tanto el daño directo como el edema y la devascularización pueden ser reversibles en el tiempo, lo que explica la existencia de hipocalcemias transitorias.

Al tener la paratohormona (PTH) una vida media corta la disminución en la cantidad de PTH liberada al plasma debido a alguno de los mecanismos de injuria antes enunciados, implica que la cantidad circulante de ésta disminuya en forma rápida. El nadir de PTH se alcanza entre las 4 y $6 \mathrm{~h}^{8,13}$. Pese a esto, los niveles de calcio se mantienen estables por un tiempo mayor. La hipocalcemia se manifiesta más frecuentemente entre las 24 y $48 \mathrm{~h}$ posterior a la cirugía ${ }^{5,69,14,18}$. Los niveles plasmáticos de calcio pueden descender incluso hasta el cuarto día postoperatorio $^{1,7,10,19}$. La tetania se puede alcanzar varios días post-operatorios ${ }^{13}$.

El objetivo del presente trabajo es estudiar si la medición de PTH a las $6 \mathrm{~h}$ del post-operatorio de tiroidectomía total es un test sensible y preciso como indicador, que permita predecir qué pacientes se encuentran en riesgo de presentar hipocalcemia posterior a una tiroidectomía total. Los objetivos secundarios del trabajo fueron analizar qué factores relacionados a la tiroidectomía total son factores de riesgo para desarrollar hipocalcemia.

\section{Sujetos y Método}

\section{Diseño del estudio}

Serie de casos prospectiva con seguimiento.

\section{Población del estudio}

Se enroló a todos los pacientes sometidos a una tiroidectomía total, independiente de su causa, entre noviembre de 2006 y abril de 2008 en el Hospital de la Fuerza Aérea de Chile. El tiempo de seguimiento mínimo fue de 6 meses. 


\section{Protocolo del estudio}

Se midió los niveles de PTH a las $6 \mathrm{~h}$ posterior a tiroidectomía total en todos los pacientes intervenidos, desde el cierre de la piel. Criterio de exclusión fue lobectomía tiroidea. La PTH fue medida con la máquina Elecsys 2010 (Roche Diagnostics, Manheim, Alemania). El rango normal para esta técnica es entre 15 y $65 \mathrm{pg} / \mathrm{mL}$ y se tomó como nivel de corte el valor normal bajo. Se registraron datos epidemiológicos relevantes (sexo, edad y diagnóstico de pre-operatorio), factores relacionados con la cirugía (cambio en el diagnóstico durante la cirugía, disección cervical, visualización de las paratiroides, autotrasplante de paratiroides y tiempo operatorio) y datos de la evolución post-operatoria (nivel de PTH a las $6 \mathrm{~h}$ expresado en $\mathrm{pg} / \mathrm{dL}$, manifestación clínica de hipocalcemia al primer, segundo y tercer día, días de hospitalización, uso de suplementos de calcio y vitamina D y si la hipocalcemia fue transitoria o no). El cambio en el diagnóstico intra-operatorio se hizo con biopsia rápida. Los signos y síntomas registrados fueron parestesias, calambres, mioclonías, signo de Chvostek y signo de Trousseau.

\section{Variables en estudio}

La variable en estudio fue el desarrollo de hipocalcemia sintomática.

\section{Definiciones}

Se definió hipocalcemia sintomática aquella que presentó síntomas y signos asociados a calcemia baja. Como hipocalcemia transitoria se definió la que requirió suplementar calcio por menos de 6 meses. El protocolo fue aprobado sin observaciones por el comité de ética del hospital, al no intervenir con la evolución esperada del paciente y estudiar una variable bioquímica. Se realizó consentimiento informado en todos los pacientes.

\section{Plan de análisis}

Se diseñó una hoja computacional de recolección de datos donde se ingresaron las variables clínicas, de laboratorio y evolución de los pacientes. El análisis de los datos se hizo en el software STATA 10.1/ $\mathrm{SE}^{\circledR}$. Se determinó mediante $\chi^{2}$ la asociación entre niveles de PTH bajo 15 pg/dL e hipocalcemia sintomática. Se calculó el riesgo relativo de presentar hipocalcemia sintomática en sujetos que presentaron PTH bajo lo normal a las $6 \mathrm{~h}$ de la cirugía y sus intervalos de confianza. Al comparar variables continuas se utilizó el test " $\mathrm{t}$ " de Student y la dócima de proporciones para comparar tasas. Se analizó a través de un análisis univariado y multivariado cuales son los factores asociados a hipocalcemia sintomática.

\section{Resultados}

En el período estudiado fueron intervenidos 82 pacientes. La distribución por sexo fue 65 (79,3\%) mujeres y $17(20,7 \%)$ hombres. La edad promedio fue de 53,2 años con un rango entre 15 y 81 años. Dos pacientes $(2,4 \%)$ presentaban bocio intratorácico. En 13 pacientes $(15,9 \%)$ existía el diagnóstico preoperatorio de cáncer de tiroides comparado con $31(37,8 \%)$ de las biopsias posteriores a la cirugía, lo que da una diferencia significativa $(p=0,0015)$. En 17 enfermos (20,7\%) hubo cambio intra-operatorio de diagnóstico a cáncer. En 10 pacientes $(12,2 \%)$ se realizó algún tipo de disección cervical, 7 (8,5\%) de los cuales fueron específica del grupo VI. En 68 pacientes $(82,9 \%)$ se visualizó al menos una paratiroides. En 13 pacientes $(15,9 \%)$ se reimplantó una paratiroides. El tiempo operatorio promedio fue 114 min con rango entre 60 y $335 \mathrm{~min}$. Sólo 19 pacientes $(23,2 \%)$ presentaron hipocalcemia sintomática, 18 de ellos esta fue transitoria. Sólo 1 paciente $(1,2 \%)$ quedó con hipocalcemia definitiva y correspondió a un paciente con cáncer de tiroides con extensión intratorácica operado con cervicotomía y esternotomía. El promedio de la calcemia para estos pacientes fue de 7,45 mg/dL con un rango de 6 a $8,1 \mathrm{mg} / \mathrm{dL}$. El valor promedio de PTH a las $6 \mathrm{~h}$ post-operatorias fue $28,71 \mathrm{pg} / \mathrm{dL}$ con rango entre 3,3 y 80,3 pg/dL. En los controles alejados se descartó hiperparatiroidismo en dos pacientes con valores supranormales. En 27 pacientes $(32,9 \%)$ se suplementó calcio oral y en 21 $(25,6 \%)$ se asoció vitamina $D$ en su forma de 1,25 -dihiroxicolecalciferol (Rocaltrol ${ }^{\circledR}$ ) (Tabla 1).

En 32 pacientes (39\%) se detectó una PTH menor de $15 \mathrm{pg} / \mathrm{dL}$. La Tabla 2 muestra la distribución entre una PTH bajo su valor normal e hipocalcemia. La asociación entre estos dos parámetros fue estadísticamente significativa $(p<0,0001)$. El riesgo relativo de presentar hipocalcemia si la PTH a las $6 \mathrm{~h}$ de la cirugía es menor a $15 \mathrm{pg} / \mathrm{dL}$ es de 4,84 (IC 1,98$7,86)$. La sensibilidad del test fue $100 \%$, su especificidad fue $79,36 \%$, el valor predictivo positivo fue $59,37 \%$ y el valor predictivo negativo fue $100 \%$. La precisión global del test fue 84,14\%. El likelihood ratio negativo fue 0 (Tabla 3 ).

El análisis univariado, controlado por edad y sexo, demostró que los factores asociados a hipocalcemia son el diagnóstico pre-operatorio de cáncer $(p=0,01$; IC 95\% 0,41-3,07), el vaciamiento cervical central $(p=0,03$; IC 95\% 0,13-3,16) y el nivel de parathormona a las $6 \mathrm{~h}$ de post-operado $(\mathrm{p}=0,002$; IC $95 \%-0,33-0,79)$.

El análisis multivariado demostró que sólo nivel de parathormona medido a las $6 \mathrm{~h}$ se asocia con hipocalcemia ( $p=0,002$; IC 95\% -0,37 -0,08) (Tabla 4). 
Tabla 1. Resumen de estadísticas

\begin{tabular}{|c|c|c|c|c|}
\hline Variable & Normocalcemia & Hipocalcemia & Todos & p value \\
\hline \multicolumn{5}{|l|}{ Datos demográficos } \\
\hline Edad & 54,7 & 48,1 & 53,2 & 0,1673 \\
\hline Sexo femenino & $84,2 \%$ & $77,8 \%$ & $79,3 \%$ & 0,5443 \\
\hline Diagnóstico pre-operatorio de cáncer & $9,5 \%$ & $36,8 \%$ & $15,9 \%$ & 0,004 \\
\hline \multicolumn{5}{|l|}{ Cirugía } \\
\hline Cambio de diagnóstico intra-operatorio & $22,2 \%$ & $15,8 \%$ & $20,7 \%$ & 0,5443 \\
\hline Vaciamiento cervical & $7,9 \%$ & $26,3 \%$ & $12,2 \%$ & 0,0319 \\
\hline Visualización de paratiroides & $84,1 \%$ & $78,9 \%$ & $82,9 \%$ & 0,5989 \\
\hline Autotrasplante de paratiroides & $12,7 \%$ & $26,3 \%$ & $15,9 \%$ & 0,1543 \\
\hline Tiempo operatorio & 115 & 109 & 114 & 0,5400 \\
\hline Días de hospitalización & 3,5 & 3,6 & 3,5 & 0,9246 \\
\hline PTH & 34,9 & 8,1 & 28,7 & $<0,0001$ \\
\hline
\end{tabular}

Unidades para tiempo operatorio $=$ minutos y para PTH pg/dL.

Tabla 2. Tabla $2 \times 2$

Tabla 3. Análisis derivado de tabla 2 × 2

\begin{tabular}{|lccc|}
\hline Variable & $\begin{array}{c}\text { Hipocal- } \\
\text { cemia }\end{array}$ & $\begin{array}{c}\text { Normo- } \\
\text { calcemia }\end{array}$ & Total \\
\hline $\mathrm{PTH}<15 \mathrm{pg} / \mathrm{dL}$ & 19 & 13 & 32 \\
$\mathrm{PTH}>15 \mathrm{pg} / \mathrm{dL}$ & 0 & 50 & 50 \\
Total & 19 & 63 & 82 \\
\hline
\end{tabular}

\begin{tabular}{|lc|}
\hline Variable & Valor \\
\hline Sensibilidad & $100 \%$ \\
\hline Especificidad & $79,4 \%$ \\
\hline Valor predictivo positivo & $59,4 \%$ \\
\hline Valor predictivo negativo & $100 \%$ \\
\hline Precisión & $84,1 \%$ \\
\hline Likelihood ratio negativo & 0 \\
\hline
\end{tabular}

Tabla 4. Análisis multivariado

\begin{tabular}{|lcccc|}
\hline Variable & Coeficiente & \multicolumn{2}{c|}{ Intervalo de Confianza 95\% } \\
Bajo & Alto & p value \\
\hline Edad & $-0,018$ & $-0,662$ & 0,028 & 0,439 \\
Sexo Femenino & 0,952 & $-1,397$ & 3,302 & 0,427 \\
Diagnóstico pre-operatorio de cáncer & 3,089 & $-0,864$ & 7,043 & 0,126 \\
Disección cervical & $-2,212$ & $-6,596$ & 2,170 & 0,322 \\
PTH & $-0,226$ & $-0,372$ & $-0,08$ & 0,002 \\
\hline
\end{tabular}

\section{Discusión}

Nuestro estudio demuestra que la medición de PTH a las 6 h de una tiroidectomía total es un método eficaz para separar dos grupos de pacientes: uno que permanecerá asintomático y otro que tiene elevado riesgo de hipocalcemia sintomática. Un valor de $\mathrm{PTH}<$ a $15 \mathrm{pg} / \mathrm{dL}$ es predictor de riesgo de hipocalcemia con una sensibilidad y valor predictivo negativo de $100 \%$ y un likelihood ratio negativo de
0 . Valores de PTH $>15 \mathrm{pg} / \mathrm{mL}$, en cambio, no se asociaron a hipocalcemia en el seguimiento.

El análisis multivariado confirmó que el único factor que permite predecir hipocalcemia es el nivel de PTH a las $6 \mathrm{~h}$ posterior a la tiroidectomía total. Limitación de este estudio es que no se midió la calcemia en forma seriada y sólo se evaluaron los síntomas; lo cual al fin y al cabo es lo que nos preocupa.

La hipocalcemia es la complicación más frecuente de la tiroidectomía total. En el presente estudio, 
la tasa de hipocalcemia, tanto transitoria $(22 \%)$ como definitiva $(1,2 \%)$, se ajusta a lo descrito en la literatura internacional. Sólo hubo un caso de hipoparatiroidismo permanente en esta serie que correspondió a un paciente que presentaba un carcinoma papilar de gran tamaño que comprometía mediastino posterior y al nervio laríngeo recurrente derecho, $\mathrm{y}$, en el que su cirugía se realizó una disección radical mediante esternotomía y no pudo preservarse ninguna paratiroides.

Algunos autores plantean factores de riesgo asociados a hipocalcemia post-operatoria tales como completar una tiroidectomía total en un paciente previamente operado de una lobectomía tiroidea debido a la distorsión de la anatomía y el difícil reconocimiento de las paratiroides ${ }^{6,19}$, la cirugía por cáncer de tiroides ${ }^{4}$ y la disección cervical del grupo $\mathrm{VI}^{19}$. Otros autores estiman que ni la cirugía por cáncer ni por enfermedad de Graves son factores de riesgo en el desarrollo de hipocalcemia ${ }^{6,16}$. También existen reportes que indican que la disección del Grupo VI no aumenta el riesgo de hipocalcemia ${ }^{14}$. Raramente es posible saber qué factores pueden predecir hipocalcemia tanto en el pre como en el intra-operatorio dado que no existen elementos que sean confiables para este $f^{5}{ }^{5,13}$. No se ha demostrado que la identificación y preservación de las 4 paratiroides prevenga la hipocalcemia ${ }^{1}$. El hecho de preservar al menos una de estas glándulas podría mantener una función paratiroidea normal y en caso de que esta disminuyera incluso podría recuperarse ${ }^{8}$. La experiencia del cirujano y el autotrasplantar las glándulas paratiroideas removidas podrían disminuir la incidencia de hipocalcemia definitiva ${ }^{14}$. De esta forma tener una herramienta efectiva en predecir hipocalcemia nos permite dar alta a las $24 \mathrm{~h}$ suplementando con calcio oral sólo a los pacientes que lo necesitan ${ }^{20,21}$. El valor del trabajo está en valor predictivo negativo de $100 \%$. Es decir, que ningún paciente tuvo hipocalcemia con valores normales de PTH. De esta forma todos los pacientes que van a hacer hipocalcemia son identificados sin excepción.

$\mathrm{Al}$ analizar por grupo a los pacientes que desarrollaron hipocalcemia sintomática versus los que se mantuvieron asintomáticos, se observa mayor frecuencia del trastorno en los pacientes con diagnóstico pre-operatorio de cáncer y los sometidos a disección cervical central. Esto ha sido sugerido por algunos autores ${ }^{1,6,8,15}$. Al igual que en otras series ni la visualización y preservación de las paratiroides ni el autotrasplante de ellas sirvió para prevenir hipocalcemia post-operatoria ${ }^{8}$.

Esta forma de predecir hipocalcemia clínica con la medición de PTH a las $6 \mathrm{~h}$ postoperatoria ha sido incorporada al manejo estándar de nuestros pacientes, confirmando con la experiencia lo acertado da la predicción y tamizando a los pacientes en forma correcta, para identificar aquellos pacientes que van a requerir suplementación de calcio y permitir así alta precoz (a las $24 \mathrm{~h})$.

\section{Referencias}

1. Grodski S, Farrell S. Early Postoperative PTH Levels as a Predictor of Hypocalcaemia and Facilitating Safe Early Discharge After Total Thyroidectomy. Asian J Surg. 2007;30:178-82.

2. Chia S, Weisman R, Tieu D, Kelly C, Dillmann W, Orloff L, et al. Prospective Study of Perioperative Factors Predicting Hypocalcemia After Thyroid and Parathyroid Surgery. Arch Otolaryngol Head Neck Surg. 2006;132:41-5.

3. Sywak M, Palazzo F, Yeh M, Wilkinson M, Snook K, Sidhu S, et al. Parathyroid Hormone Assay Predicts Hypoclacemia After Total Thyroidectomy.ANZ J Surg. 2007;77:667-70.

4. Payne R, Tewfik M, Hier M, Tamilia M, Mac Namara E, Young J, et al. Benefits Resulting from 1-and 6-hour Parathyroid Hormone and Calcium Levels After Thyroidectomy. Otolaryngol Head Neck Surg. 2005;133:38690.

5. Lombardi C, Raffaelli M, Princi P, Dobrinja C, Carrozza C, Di Stasio E, et al. Parathyroid hormone levels 4 hours after surgery do not accurately predict post-thyroidectomy hypocalcemia. Surgery 2006;140:1016-25.

6. Scurry C, Beus K, Hollenbeak S, Stack B. Perioperative Parathyroid Hormone Assay for Diagnosis and Management of Postthyroidectomy Hypocalcemia. Laryngoscope 2005;115:1362-66.

7. Pattou F, Combemale F, Fabre S, Carnaille B, Decoulx M, Wemeau JL, et al. Hypocalcemia following Thyroid Surgery: Incidence and Prediction of Outcome. World J Surg. 1998;22:718-24.

8. Kihara M, Miyauchi A, Kontani K, Yamauchi A, Yokomise H. Recovery of Parathyroid Function afer Total Thyroidectomy: Long-Term Follow-Up Study. ANZ J Surg. 2005;75:532-36.

9. Noordzij P, Lee S, Bernet V, Payne R, Cohen S, McLeod I, et al. Early Prediction of Hypocalcemia after Thyroidectomy using Parathyroid Hormone: An Analysis of Pooled Individual Patient Data from Nine Observational Studies. J Am Coll Surg. 2007;205:748-54.

10. Soon P, Magarey C, Campbel P, Jalaludin B. Serum Intact Parathyroid Hormonas, a Predictor of Hypocalcaemia after Total Thyroidectomy. ANZ J Surg. 2005; 75:977-80.

11. Vescan A, Witterick I, Freeman J. Parathyroid Hormone as a Predictor of Hypocalcemia after Thyroidectomy. Laryngoscope 2005;115:2105-8.

12. Potts J. Diseases of the Parathyroid Glands and Other Hyper-and Hypocalcemic Disorders. En: Braunwald E, 
Fauci A, Kasper D, Hauser S, Longo D, Jameson J, Editores, Harrison's Principles of Internal Medicine 15th Edition. EEUU: Editorial Mc Graw Hill Companies, Inc.; 2001. p. 2205-2225.

13. Lam A, Kerr P. Parathyroid Hormone: An Early Predictor of Postthyroidectomy Hypocalcemia. Laryngoscope 2003;113:2196-200.

14. Lombardi C, Raffaelli M, Princi P, Santini S, Boscherini $\mathrm{M}$, De Crea C, et al. Early prediction of postthyroidectomy hypocalcemia by one single iPTH measurement. Surgery 2004;136:1236-41.

15. Asari R, Passler C, Kaczirek K, Scheuba C, Niederle B. Hypoparathyroidism After Total Thyroidectomy. Arch Surg. 2008;143:132-7.

16. Warren F, Andersen P, Wax M, Cohen J. Perioperative Parathyroid Hormone Levels in Thyroid Surgery: Preliminary Report. Laryngoscope 2004;114:689-93.

17. Del Río P, Arcuri MF, Ferreri G, Sommaruga L, Sianesi M. The utility of serum PTH assessment 24 hours after total thyroidectomy. Otolaryngol Head Neck Surg. 2005;132:584-6.

18. Grodski S, Campbell P, Cook MJ, Delbridge L, Farrell S, Gough I, et al. Australian Endocrine Surgeons Guidelines AES06/01. Postoperative Parathyroid Hormone Measurementand Early Dischargeafter Total Thyroidectomy: Analysis of Australian Data and Management Recommendation. ANZ J Surg. 2007;77:199-202.

19. Grodski S, Serpell J. Evidence for the Role of Perioperative PTH Measurement after Total Thyroidectomy as a Predictor of Hypocalcemia. World J Surg. 2008;32:1367-73.

20. Ghaheri B, Liebler S, Andersen P, Schuff K, Samuels M, Klein R, et al. Perioperative Parathyroid Hormone Levels in Thyroid Surgery. Laryngoscope 2006;116:518-21.

21. Lindblom P, Westerdahl J, Bergenfelz A. Low parathyroid hormone levels after thyroid surgery: A feasible predictor of hypocalcemia. Surgery 2002;131:51520. 